\title{
Identified charged particle azimuthal anisotropy in PHENIX at RHIC
}

\author{
ShinIchi Esumi, ${ }^{\text {a }}$ for the PHENIX collaboration * \\ anst. of Physics, Univ. of Tsukuba, Tenno-dai 1-1-1, Tsukuba, Ibaraki 305, Japan
}

Event anisotropy is expected to have sensitivity to the early stage of ultra-relativistic heavy-ion collisions at RHIC. The possible formation of a quark gluon plasma (QGP) could affect how the initial anisotropy in the space coordinate is transferred into the momentum space for the final state. The anisotropy parameter $\left(\mathrm{v}_{2}\right)$ is an amplitude of the 2nd harmonic parameter of the azimuthal distribution with respect to the reaction plane. We present here $\mathrm{v}_{2}$ of identified and inclusive charged particles measured in the PHENIX central arm detector $(|\eta|<0.35)$ with respect to the reaction plane defined at $|\eta|=3 \sim 4$ in $200 \mathrm{GeV} \mathrm{Au}+\mathrm{Au}$ collisions. We find that $\mathrm{v}_{2}$ increases from central to midcentral collisions reaching a maximum at about $50 \%$ of the geometric cross section and then decreases again for more peripheral collisions. As a function of transverse momentum in minimum-bias collisions, the $\mathrm{v}_{2}$ parameter increases linearly with $\mathrm{p}_{\mathrm{T}}$ up to $\mathrm{p}_{\mathrm{T}} \simeq 2$ $\mathrm{GeV} / \mathrm{c}$ and then saturates for inclusive charged particles. The $\mathrm{v}_{2}$ parameter of identified particles $\left(\pi^{+}, \pi^{-}, K^{+}, K^{-}, p\right.$ and $\left.\bar{p}\right)$ follow a hydro-dynamic behavior up to $2 \mathrm{GeV} / \mathrm{c}$ in $\mathrm{p}_{\mathrm{T}}$, where the lighter mass particles have larger $\mathrm{v}_{2}$ at a given $\mathrm{p}_{\mathrm{T}}$. However there is an indication that this trend is reversed at around $\mathrm{p}_{\mathrm{T}} \simeq 2 \mathrm{GeV} / \mathrm{c}$, where $p$ and $\bar{p}$ have larger $\mathrm{v}_{2}$ than $\pi$ and $K$.

\section{Introduction and Experimental Setup}

The PHENIX experiment at RHIC is designed to measure a variety of observables in ultra-relativistic heavy-ion collisions. The primary goal is to detect signals of the QGP. The $\mathrm{v}_{2}$ measurements [1], 2] at RHIC energies have been performed in several experiments for inclusive charged particles and for identified particles. Extending the $\mathrm{v}_{2}$ measurements at higher $\mathrm{p}_{\mathrm{T}}$ region, especially with identified particles, is important to understand the origin of the $\mathrm{v}_{2}$ component: hydro-dynamic flow of compressed matter, the production of many mini-jets, etc. It has also been observed that $\mathrm{v}_{2}$ saturates at $\mathrm{p}_{\mathrm{T}} \sim 2 \mathrm{GeV} / \mathrm{c}$. The cause of this is not yet known, but it is worth noting that at this momentum the particle composition is very different than at low momentum in that the proton yield is comparable to the pion yield [3].

PHENIX has excellent particle identification at mid-rapidity. In this analysis, the time-of-flight (TOF) detector is used to identify final state hadrons up to $4 \mathrm{GeV} / \mathrm{c}$ in $\mathrm{p}_{\mathrm{T}}$ together with the central arm tracking system consisting of drift- and pad-chambers for momentum reconstruction. The beam counters (BBC) provide collision time and z-vertex

*for the full PHENIX Collaboration author list and acknowledgements, see Appendix "Collaborations" of this volume. 
position information. The two beam counters are located at $|z|=1.5 \mathrm{~m}$ from the collision point, surrounding the beam pipe with 64 photo-multiplier tubes, covering $|\eta|=3 \sim 4$. The large charged multiplicity at $|\eta|=3 \sim 4$ and non-zero signal of event anisotropy in this $\eta$ range enables us to measure the event reaction plane using the BBC with full azimuthal angle coverage. The standard method [ 4, 5] for calculating and correcting (flattening) the reaction plane is applied here. In order to obtain an improved reaction plane resolution, a combined reaction plane is defined by averaging the reaction plane angles obtained from each BBC using the elliptic moment. The estimated resolution of the combined reaction plane, $<\cos 2\left(\Phi_{\text {measured }}-\Phi_{\text {truth }}\right)>$, is an average of 0.3 with a maximum of about 0.4 .

\section{Results and Interpretation}

Charged particles are measured in the central arm spectrometers $(|\eta|<0.35)$. In the present analysis, the $\mathrm{p}_{\mathrm{T}}$ range of the particle identification is $0.2<\mathrm{p}_{\mathrm{T}}<3 \mathrm{GeV} / \mathrm{c}$ for pions, $0.3<\mathrm{p}_{\mathrm{T}}<2 \mathrm{GeV} / \mathrm{c}$ for kaons, and $0.5<\mathrm{p}_{\mathrm{T}}<4 \mathrm{GeV} / \mathrm{c}$ for protons. The centrality of the collision is defined by the correlation between the total number of particles measured in the BBC and the total energy measured in zero degree calorimeter.

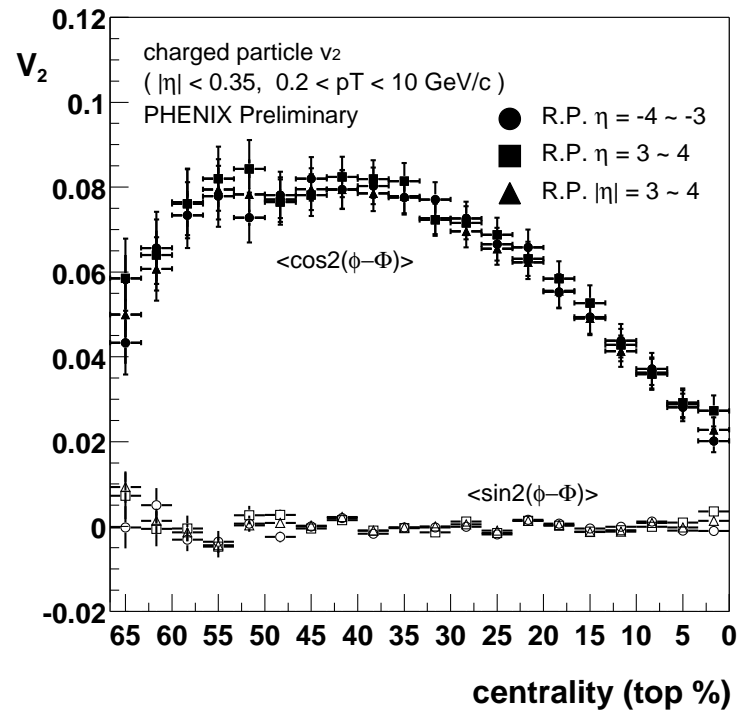

Figure 1. Centrality dependence of $\mathrm{v}_{2}$ for inclusive charged particle measured at midrapidity $(|\eta|<0.35)$ and $\mathrm{p}_{\mathrm{T}}$ integrated between 0.2 and $10 \mathrm{GeV} / \mathrm{c}$ with respect to the reaction plane defined by the two beam counters separately or combined. Here R.P. stands for the reaction plane within the specified $\eta$ range.

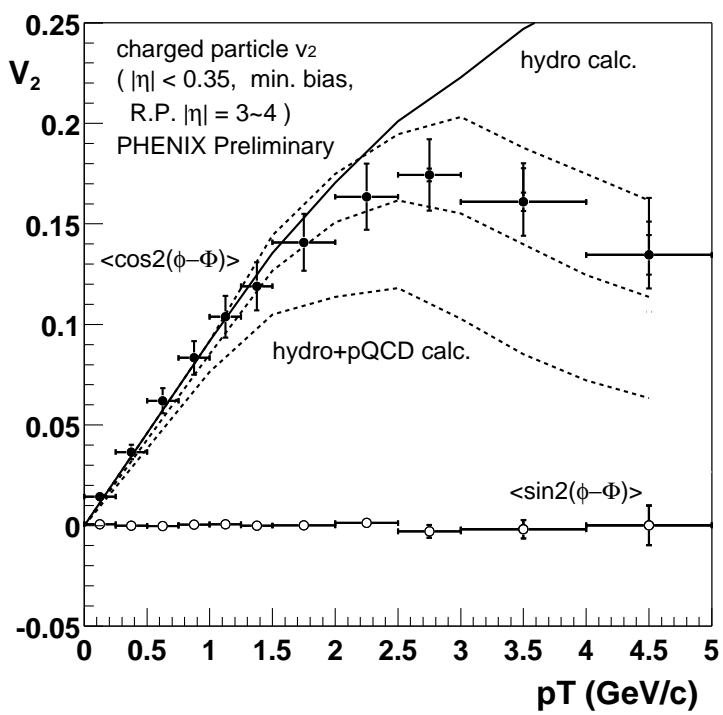

Figure 2. Transverse momentum dependence of $\mathrm{v}_{2}$ for inclusive charged particles at mid-rapidity $(|\eta|<0.35)$ with respect to the combined reaction plane $(|\eta|=3 \sim 4)$ for minimum-bias collisions. Included are comparisons to the model calculations, where the details are described in the text below and in refs [6, 7]. 
Figure 1 shows the centrality dependence of $\mathrm{v}_{2}$ for inclusive charged particles measured at mid-rapidity $(|\eta|<0.35)$ with respect to three different reaction planes: two determined using each beam counter separately, and one determined from the combination of the two beam counters. Here R.P. stands for the reaction plane within the $\eta$ range shown in the figure. The three results agree well, indicating that the resolution for the different reaction planes is well understood. The $\mathrm{v}_{2}$ parameter decreases for both peripheral and central collisions with a maximum at about $50 \%$ of the geometric cross section. The reduction of $\mathrm{v}_{2}$ for peripheral events might indicate that non-flow contributions are less significant in this analysis because of the large rapidity gap between the reaction plane and the central tracking arm acceptance of about 3 units. The data are consistent with a $<\sin 2(\phi-\Phi)>$ distribution (represented by the open symbols), which is expected to be zero. Figure 2 shows the transverse momentum dependence of $\mathrm{v}_{2}$ for inclusive charged particles with respect to the combined reaction plane for minimum-bias events. The data above a $\mathrm{p}_{\mathrm{T}}$ of $2 \mathrm{GeV} / \mathrm{c}$ clearly show a deviation from the linear behavior seen at smaller $\mathrm{p}_{\mathrm{T}}$. The solid line is from a hydro-dynamic calculation [ [6]. The three dashed lines are from a hydro + pQCD calculation with three different gluon densities (from top to bottom, $\mathrm{dN}^{\mathrm{g}} / \mathrm{dy}=$ 1000, 500, and 200) [7].
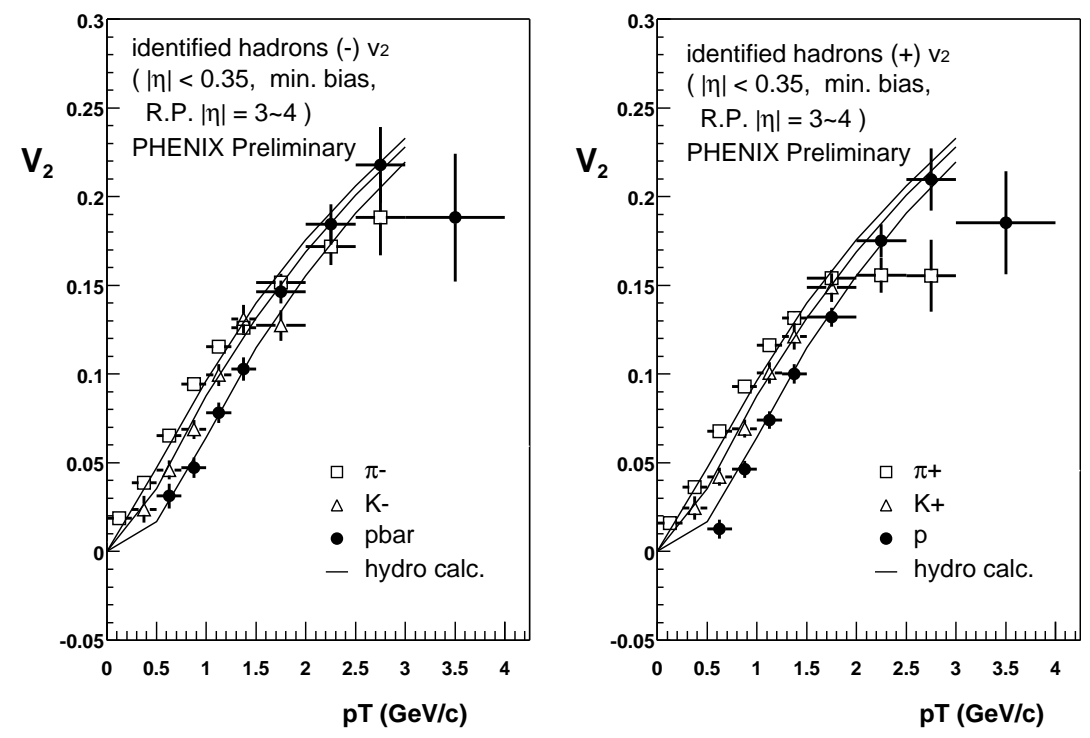

Figure 3. Transverse momentum dependence of $\mathrm{v}_{2}$ for identified particles, $\pi^{-}$, $K^{-}, \bar{p}$ (left) and $\pi^{+}, K^{+}$, $p$ (right). The solid lines represent a hydro-dynamic calculation [6] including a first-order phase transition with a freeze out temperature of $120 \mathrm{MeV}$ for $\pi, K$ and $p$ from upper to lower curves, respectively.

Figure 3, the transverse momentum dependence of $\mathrm{v}_{2}$ for identified particles is shown. The solid circles show $p$ and $\bar{p}$, the open triangles show $K^{+}$and $K^{-}$, and the open squares show $\pi^{+}$and $\pi^{-}$. The left panel shows negatively charged particles, while the right panel shows positively charged particles. The solid lines represent hydro-dynamic calculation [ [6] including a first-order phase transition with a freeze out temperature of $120 \mathrm{MeV}$. The data show that at lower $\mathrm{p}_{\mathrm{T}}(<2 \mathrm{GeV} / \mathrm{c})$ the lighter mass particles have a larger $\mathrm{v}_{2}$ at a given $\mathrm{p}_{\mathrm{T}}$, which is reproduced by the model calculations. However, the data and the calculation deviate at higher $\mathrm{p}_{\mathrm{T}}$. Lighter particles seem to deviate from the hydro-dynamic behavior at lower $\mathrm{p}_{\mathrm{T}}$ than for the heavier particles. Consequently, the mass dependence of $\mathrm{v}_{2}$ for $\mathrm{p}_{\mathrm{T}}$ above $2 \mathrm{GeV} / \mathrm{c}$ is reversed. Since the particle identification capability for $K / p$ separation goes up to $4 \mathrm{GeV} / \mathrm{c}$, the combined $\pi$ and $K$ can be compared with protons up to $4 \mathrm{GeV} / \mathrm{c}$. The $\mathrm{v}_{2}$ value from the combined $\pi$ and $K$ are shown in figure 4 in order to 

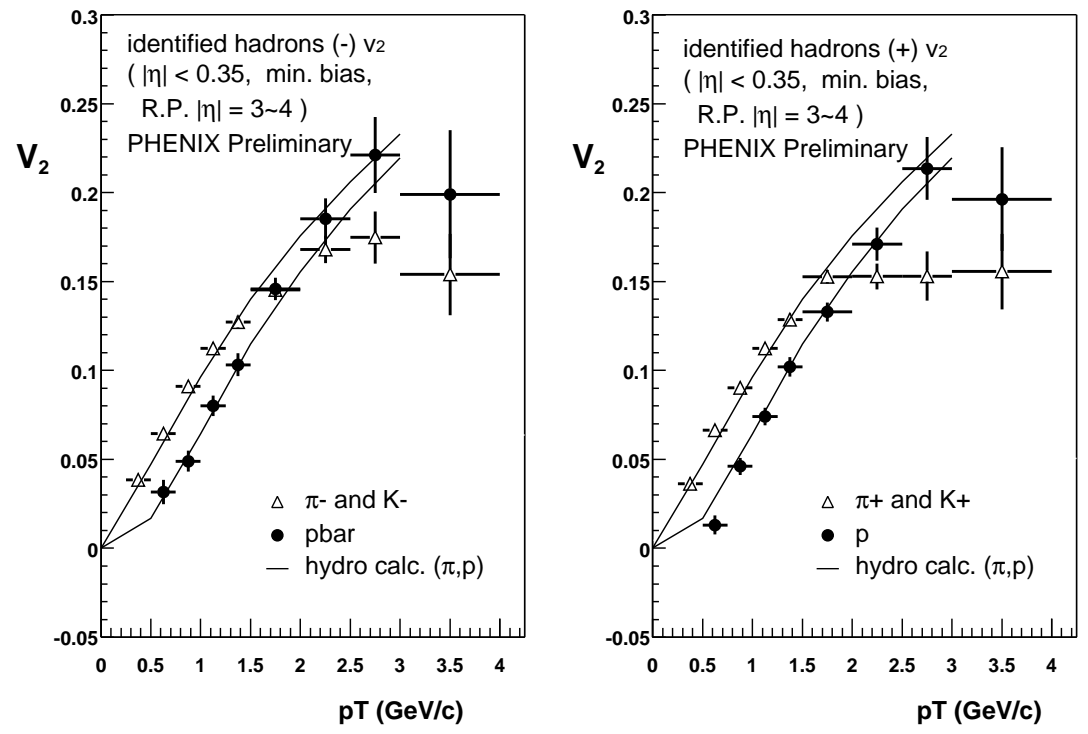

Figure 4. Transverse momentum dependence of $\mathrm{v}_{2}$ for $\pi^{-}$and $K^{-}$compared to $\bar{p}$ (left) and for $\pi^{+}$and $K^{+}$compared to $p$ (right). The solid lines are hydrodynamic calculation [6] for $\pi$ (upper curve) and $p$ (lower curve). A clear switch over between $\pi+K$ and proton is seen at about $\mathrm{p}_{\mathrm{T}}=2 \mathrm{GeV} / \mathrm{c}$.

clearly show their difference from the $p$ and $\bar{p}$. The reversed trend of $\mathrm{v}_{2}$ at $\mathrm{p}_{\mathrm{T}}$ above 2 $\mathrm{GeV} / \mathrm{c}$ is clearly seen.

\section{Summary}

The value of $\mathrm{v}_{2}$ for identified and inclusive charged particles are measured at midrapidity with respect to the reaction plane defined in the forward and backward rapidity regions in $200 \mathrm{GeV} \mathrm{Au}+\mathrm{Au}$ collisions in the PHENIX experiment at $\mathrm{RHIC}$. The $\mathrm{v}_{2}$ value of inclusive charged particles decreases for both peripheral and central collisions with a maximum at about $50 \%$ of the geometric cross section. The $\mathrm{v}_{2}$ value increases linearly with $\mathrm{p}_{\mathrm{T}}$ at lower $\mathrm{p}_{\mathrm{T}}$ and saturates around $2 \mathrm{GeV} / \mathrm{c}$. The $\mathrm{v}_{2}$ parameter for identified particles follows a hydro-dynamic behavior up to $2 \mathrm{GeV} / \mathrm{c}$ in $\mathrm{p}_{\mathrm{T}}$, where the lighter mass particles have larger $\mathrm{v}_{2}$ at a given $\mathrm{p}_{\mathrm{T}}$. However, there is an indication that the trend of this mass dependence reverses at higher $\mathrm{p}_{\mathrm{T}}$.

\section{REFERENCES}

1. K.H. Ackermann et al., Phys. Rev. Lett. 86, 402, (2001), Phys. Rev. Lett. 87, 182301, (2001), J.Phys. G28, 2089, (2002).

2. K. Adcox et al., nucl-ex/0204005.

3. K. Adcox et al., Phys. Rev. Lett. 88, 242301, (2002).

4. J.Barrette et al., Phys. Rev. Lett. 73, 2532, (1994), Phys. Rev. C55, 1420, (1997), Phys. Rev. C56, 3254, (1997),

5. A.Poskanzer and S.Voloshin, Phys. Rev. C58, 1671, (1998)

6. P.Huovinen, P.F.Kolb, U.W.Heinz, P.V.Ruuskanen and S.A.Voloshin, Phys. Lett. B503, 58 (2001)

7. M.Gyulassy, I.Vitev and X.N.Wang, Phys. Rev. Lett. 86, 2537, (2001) 